\title{
LPG Gas Leakage Detection \& Control System
}

\author{
Rajeshwari Salmani
}

\begin{abstract}
Home security has been a major issue where crime is increasing in case of LPG gas leakage by unknowingly occurred accidents. User can take advantage of new technology for avoiding LPG gas leakage accidents. This paper is to present such a design that can automatically detect and stop gas leakage in vulnerable premises. This technique work by controlling the leakage by closing the valve directly in case of a leakage. It controls the automated action \& device which will shutdown the electric power to the house as well as open the kitchens windows. Owner and gas agency can receive the message about Gas leakage. By using alerting message system provide the awareness about gas leakage.
\end{abstract}

Keywords--- DCmotor, GSM, Microcontroller, Realy, Solar Panel.

\section{INTRODUCTION}

Nowadays LPG accidents occur very common. The main reason of these accidents is due to the leakage of LPG. LPG consists of mixture of propane and butane which is highly flammable chemical. It is odorless gas due to which Ethanethoil is added as powerful odorant, so that leakage can be easily detected[1].

This leakage of LPG promstarts when we forget to close the main regulator valve and if the wiring of the exhaust fan is not proper then it will cause immediate explosion since it deals with the AC[1].

To overcome these sorts' of disadvantages we proposed a system which would take the control action as well as the detection action. The proposed system takes a automatic control action after the detection of LPG leak of $0.001 \%$.This automatic control action provides the closing of valve by a mechanical handle. Next by using a GSM (Global System for Mobile Communications) we are sending the message to the fire station. At last the buzzer is provided for alerting the neighbor's about the leakage.

\section{LITERATURE SURVEY}

Meenakshi Vidya proposed the leakage detection and real time gas monitoring system. In this system, the gas leakage is detected and controlled by means of exhaust fan. The level of LPG in cylinder is also continuously monitored.

K Padmapriya proposed the design of wireless LPG monitoring system. In this project, the user is alerted about the gas leakage through SMS and the power supply is turned off.
Selvapriya proposed the system in which the leakage is detected by the gas sensor and produce the results in the audio and visual forms. It provides a design approach on software as well as hardware.

L.K.Hema proposed the smart sensor technology. In this flexible reliable smart gas detection system is developed. In this, the leakage is detected and controlled by using exhaust fan.

B. D. Jolhe proposed the system in which two sensors are used for detecting the gas leakage and for monitoring the level of gas in the cylinder respectively.

Ashish Shrivastava proposed the system in which two types of gases namely LPG and CNG are detected for home safety as well for vehicles.

R.Padmapriya proposed the system which ARM7 processor and simulates using keil software to alert the user by sending SMS.

V.Ramya proposed the system that uses two different sensors for detecting the leakage and requires resetting manually after every situation.

A.Mahalingam proposed the system to meet UK occupational health and safety standards.

\section{PROMBLEMS WITH GAS LEAKAGE}

LPG may leak as a gas or a liquid. If the liquid leaks it will quickly evaporate and form a relatively large cloud of gas which will drop to the ground, as it is heavier than air. LPG vapours can run for long distances along the ground and can collect in drains or basements. When the gas meets a source of ignition it can burn or explode. Cylinders can explode if involved in a fire. LPG can cause cold burns to the skin and it can act as an asphyxiant at high concentrations.

Rajeshwari Salmani, Student, Dept of Computer science and engineering, SKSVMACET Lxameshwar, India. E-mail:rajeshwarins33@gmail.com DOI:10.9756/BIJSESC.8246 


\section{STATSTICS}

\section{Occurrences of accidents in the past}

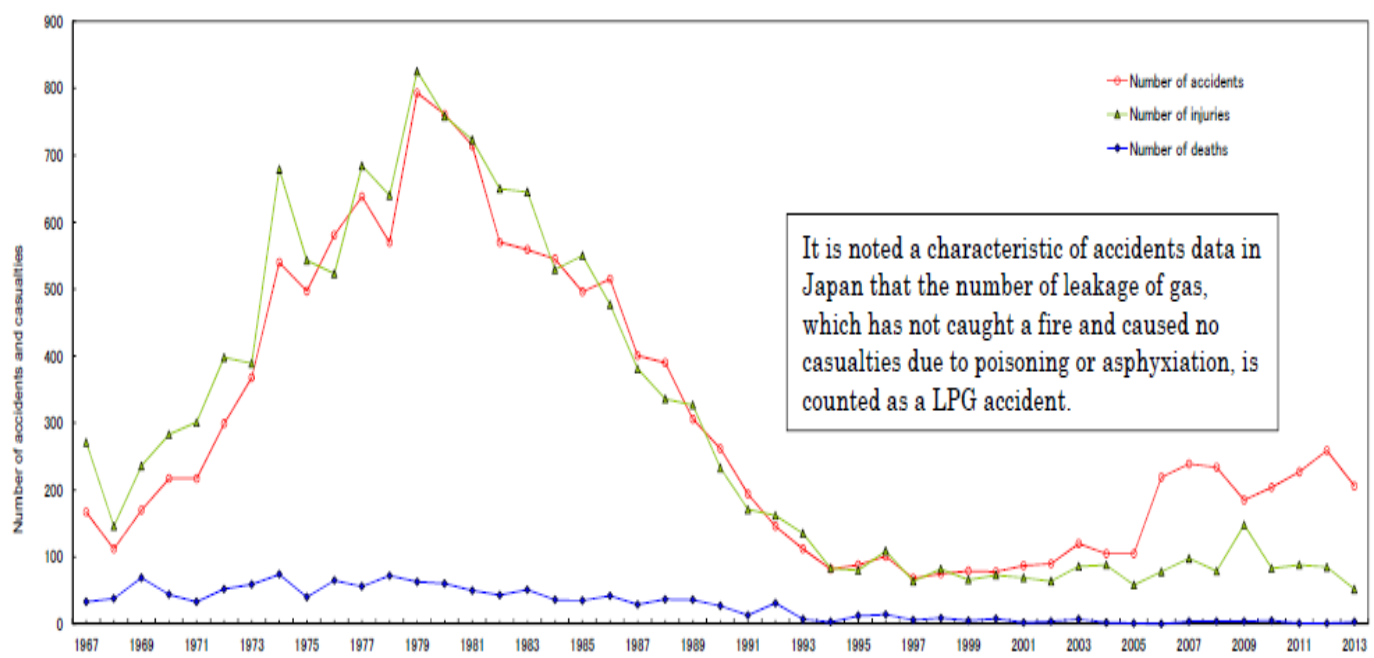

Figure 1: Statistics Of Gas Leakage

\section{Structure of Gas Leakage Detection \& CONTROL SYSTEM}

This system helps you to upgrade your safety standards, comply statutory requirements on environmental commitments and most important and basic function being prevent accidents and protect life and property from disaster.

This block diagram explain about how to detect gas leakage[2].

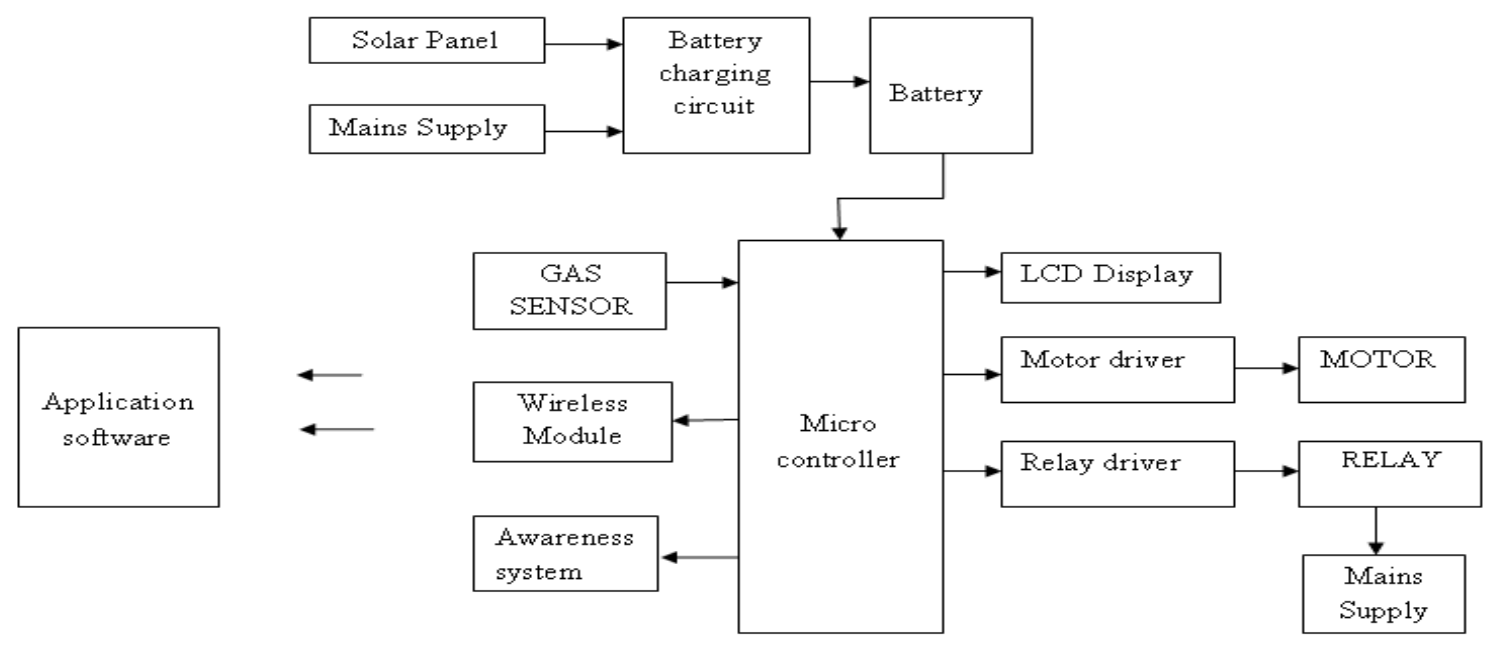

Figure 2: Block Diagram of System

\section{A. $L C D$}

LCD's are very simple to interface with the controller as well as are cost effective. The LCD requires 3 control lines (RS, R/W \& EN) \& 8 (or 4 ) data lines. The number on data lines depends on the mode of operation. If operated in 8-bit mode then 8 data lines +3 control lines i.e. total 11 lines are required. And if operated in 4-bit mode then 4 data lines +3 control lines i.e. 7 lines are require.

\section{B. GAS Sensor}

MQ-6 gas sensor modules are used in gas leakage detecting equipments in family and industry, are suitable for detecting of LPG. This is a simple-to-use liquefied petroleum gas (LPG) sensor, suitable for sensing LPG concentrations in the air .In our project use MQ-6 as gas sensor. If gas is detected by the sensor ADC calculates how much amount of gas present in the air .if it is found a larger amount microcontroller switch on relay. 


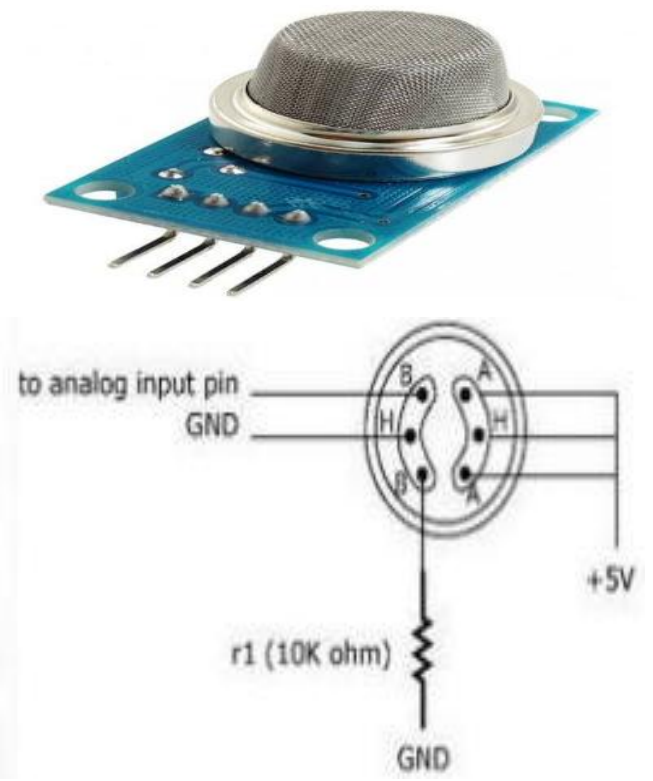

Figure 3: Gas sensor

\section{GSM Module}

Global System for Mobile Communication. Global system for mobile communication (GSM) is a globally accepted standard for digital cellular communication. GSM is the name of standardization group established in 1982 to create a common European mobile telephone standard that would formulate.

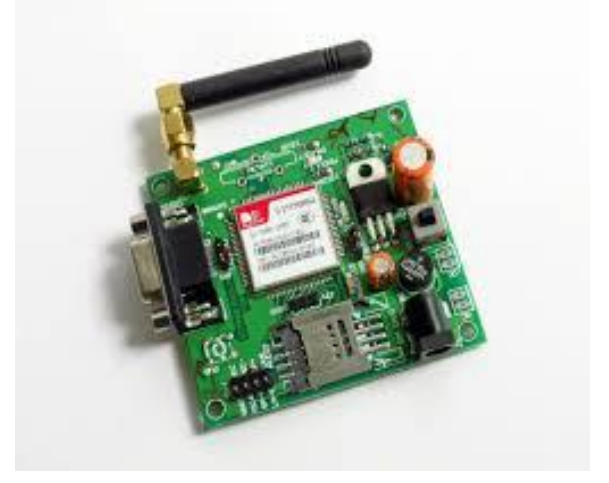

Figure 4: GSM module

Specifications for a pan-European mobile cellular radio system operating at $900 \mathrm{MHz}$. The SIM300 is a TriBand/Quad-Band GSM/GPRS solution in a compact plug-in module. The leading features of SIM300/340 make it ideal for application, such as WLL applications and handheld devices. An embedded Powerful TCP/IP protocol stack.

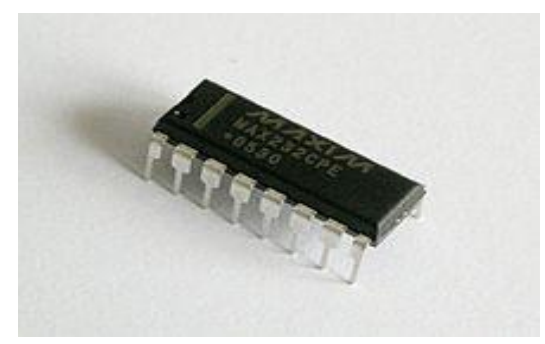

Figure 6: MAX232

\section{Relay}

Relay acts as a switch which is used to control the 230 volt AC supply. This relay will be turned off if there is no person inside the room. This relay can be used to turn off the electrical appliances like fan, tubes etc.

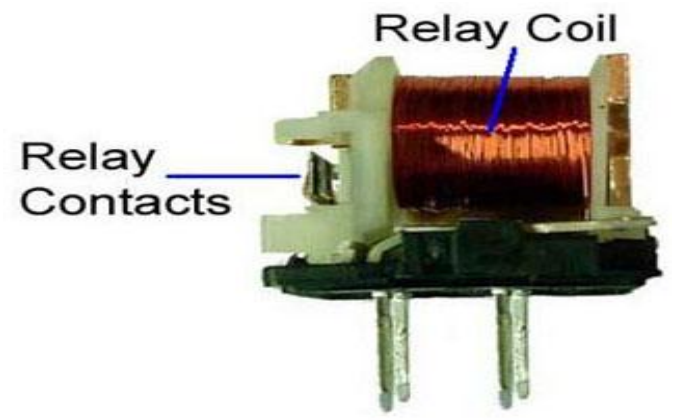

Figure 5: Relay

\section{E. Relay Driver Circuit}

Relays are components which allow a low-power circuit to switch a relatively high current on and off, or to control signals that must be electrically isolated from the controlling circuit itself. To make a relay operate, we have to pass a suitable pull-in and holding current (DC) through its energizing coil. And generally relay coils are designed to operate from a particular supply voltage, often $12 \mathrm{~V}$, in case of many small relays used for electronics work[6].

We have to provide enough base current to turn the transistor on and off.NPN transistor BC547 is used to control a Relay with a $12 \mathrm{~V}$ coil, operating from a $+12 \mathrm{~V}$ supply. Series base resistor R1 is used to set the base current for transistor, so that the transistor is driven into saturation (fully turned on) when the relay is to be energized.

\section{F. Battery}

Batteries store energy being produced by given generating source and when this source is unavailable this energy can be used by loads. The inclusion of storage in any energy generating system will increase the availability of the energy. Operating voltage of battery is $12 \mathrm{v}$ dc.

MAX232:

MAX232 IC is used for serial communication.MAX232 is compatible with RS-232 standard, and consists of dual transceiver. Each receiver converts TIA/EIA-232-E C levels into 5V TTL/CMOS levels. Each driver converts TTL/COMS levels into TIA/EIA-232-E levels[5].

\section{G. DC Motor}

DC Motors is $60 \mathrm{RPM} 12 \mathrm{~V}$ DC motors and5 $\mathrm{kg} \mathrm{cm}$ torque and No-load current $=60 \mathrm{~mA}(\mathrm{Max})$ and Load current $=300$ mA (Max).

\section{H. DC Motor Driver}

The L298 is an integrated monolithic circuit in a 15- lead Multi-watt package. It is a high voltage; high current dual fullbridge driver designed to accept standard TTL logic levels and drive inductive loads such as relays, solenoids, DC and 
stepping motors.

Two enable inputs are provided to enable or disable the device independently of the input signals.

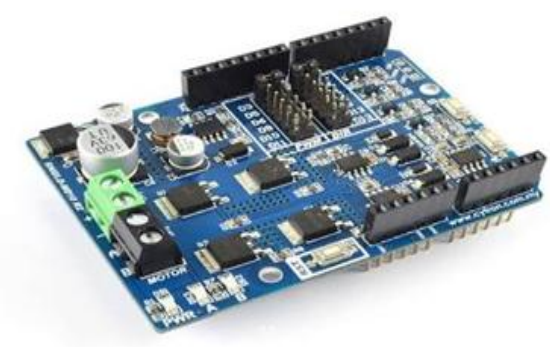

Figure 7: DC Motor Driver

\section{METHODOLOGY}

When the leakage of LPG has started, the proposed system senses the leak by means of a mq6 after sensing the sensor's output is given to the analog to digital converter then this value is given to the controller where the program is given for checking the leakage condition. The value of minimum and maximum leakage is given in terms of hexadecimal or binary form. Then this value when it is detected either as maximum or minimum value then the instruction is given to motor to open windows. Driving the motor through the driver IC. Then after controlling the leakage message is sent to fire station by means of GSM then the buzzer alert is given to the neighbour and electric power supply is shutdown by means of a relay.

\section{FLOCHART}

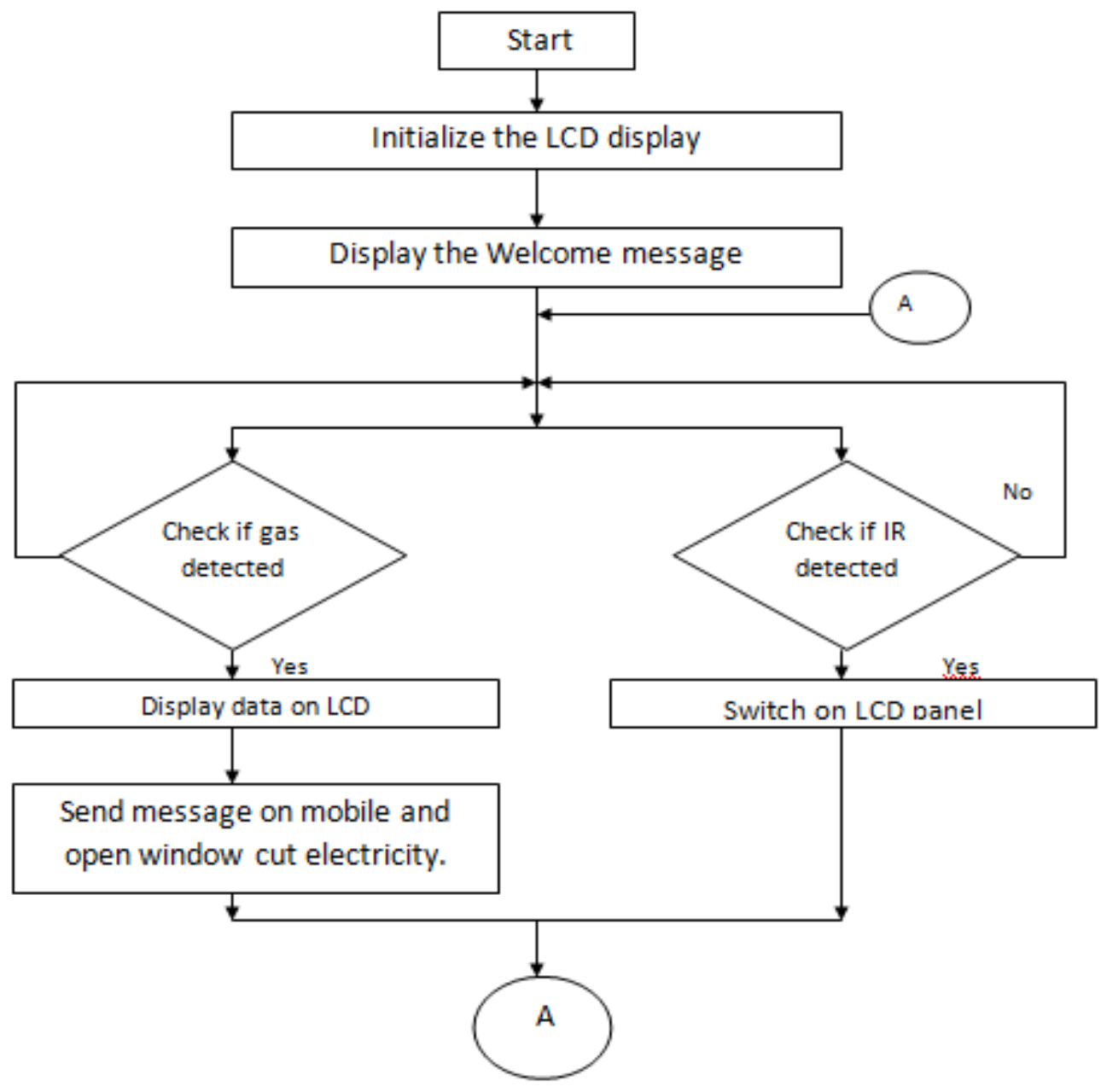

Figure 8: Flowchart for Gas Leakage Control System

\section{APPLICATION}

1. Protection from any gas leakage in home.

2. For safety from gas leakage in heating gas fired appliances.

3. Large industries which uses gas as their production.

4. For safety from gas leakage in cooking gas fired appliances like ovens, stoves etc.

5. Gas leak alarm.
IX. ADVANTAGES

1. It is used in house as LPG leakage detection.

2. The sensor has excellent sensitivity combined with a quick fast response time.

3. The system is highly reliable, tamper-proof and secure.

4. In the long run the maintenance cost is very less when Compared to the present systems. 
5. It is possible to get instantaneous results and with high accuracy.

\section{CONCLUSION}

When the gas is detected windows get opened. Also as soon as gas is detected mains are cut automatically. With this, a text message is sent to the gas agency, Government\& fire brigade. Although the system is certain amount costlier than the existing one \& it has more advantageous functions than the other systems present. This system is very easy to install and is also portable. It is more helpful in home.

\section{REFERENCES}

[1] B.K. Konstantinos Domdouzis and C. Anuba, "An experimental study of the effects of different medium on the performance of rfid system", Vol. 21, 2011.

[2] K. Finkenzeller, "Fundamentals and Applications in Contactless Smart Cards and Identification", John Wiley and Sons Ltd, 2003.

[3] Projects.org, "www.projectof 8051.com," 2011.

[4] Opensource.org,"www.alldatasheet4u.com," 2011.

[5] M. Mazidi, "8051 Microcontroller and Embedded Systems", Pear-son Education India, 2007.

[6] R.L. Boylestad and L. Nashelsky, "Electronic Devices and Circuit Theory", 8th Edition, 2006.

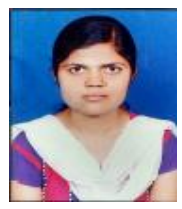

Rajeshwari Salmani, (F'20), Laxmeshwara, 03-03-1996. studying BE at SKSVMACET Laxmeshwara, Karanataka, India. (E-mail: rajeshwarins33@gmail.com ) 